\title{
Cyclic stretch promotes the ossification of ligamentum flavum by modulating the Indian hedgehog signaling pathway
}

\author{
RUI GAO ${ }^{1 *}$, CHANGGUI SHI $^{*}$, CHENGWEI YANG $^{2}$, YIN ZHAO $^{1}$, \\ $\mathrm{XIONGSHENG} \mathrm{CHEN}^{1}$ and XUHUI ZHOU ${ }^{1}$ \\ ${ }^{1}$ Department of Orthopedics, Changzheng Hospital, Second Military Medical University, Shanghai 200003; \\ ${ }^{2}$ Department of Orthopedics, Lanzhou General Hospital of PLA, Lanzhou, Gansu 730050, P.R. China
}

Received March 22, 2019; Accepted January 29, 2020

DOI: $10.3892 / \mathrm{mmr} .2020 .11200$

\begin{abstract}
The Indian hedgehog (IHH) signaling pathway is an important pathway for bone growth and development. The aim of the present study was to examine the role of the IHH signaling pathway in the development of the ossification of ligamentum flavum (OLF) at the cellular and tissue levels. The expression levels and localization of the osteogenic genes Runt-related transcription factor 2 (RUNX2), Osterix, alkaline phosphatase $(A L P)$, osteocalcin $(O C N)$ and $I H H$ were evaluated in OLF tissues by reverse transcriptionquantitative PCR (RT-qPCR) and immunohistochemistry. Non-ossified ligamentum flavum (LF) sections were used as control samples. The tissue explant method was used to obtain cultured LF cells. In addition, OLF cells were subjected to cyclic stretch application for $0,6,12$ or $24 \mathrm{~h}$. The expression levels of osteogenic genes, and the IHH signaling pathway genes $I H H$, Smoothened (SMO), GLI family zinc finger 1 (GLII), GLI2 and GLI3 were evaluated with RT-qPCR and western blotting. Osteogenic differentiation was further evaluated by assessing ALP activity and staining. Moreover, the effect of cyclopamine (Cpn), an IHH signaling inhibitor, on osteogenic differentiation was examined. The RT-qPCR
\end{abstract}

Correspondence to: Professor Xuhui Zhou or Professor Xiongsheng Chen, Department of Orthopedics, Changzheng Hospital, Second Military Medical University, 415 Fengyang Road, Shanghai 200003, P.R. China

E-mail: shanghaizhouxuhui@163.com

E-mail: cxspine@smmu.edu.cn

"Contributed equally

Abbreviations: IHH, Indian hedgehog; OLF, ossification of ligamentum flavum; RUNX2, Runt-related transcription factor 2; $A L P$, alkaline phosphatase; $O C N$, osteocalcin; LF, ligamentum flavum; Cpn, cyclopamine; OPN, osteopontin; SOX9, sex-determining region Y-box protein 9; BCA, bicinchoninic acid

Key words: ligamentum flavum, cyclic stretch, ossification, osteogenic differentiation, pathway, Indian hedgehog and immunohistochemical results indicated that the mRNA and protein expression levels of RUNX2, Osterix, $A L P, O C N$ and $I H H$ were significantly higher in the OLF group compared with the LF group. Furthermore, application of cyclic stretch to OLF cells resulted in greater ALP activity, and significant increases in mRNA and protein expression levels of RUNX2, Osterix, $A L P$ and $O C N$ in a time-d00ependent manner. Cyclic stretch application also led to significant increases in IHH signaling pathway genes, including $I H H, S M O, G L I I$ and $G L I 2$, while no significant effect was found on GLI3 expression level. In addition, it was found that Cpn significantly reversed the effect of cyclic stretch on the ALP activity, and the expression levels of RUNX2, Osterix, ALP, OCN, GLI1 and GLI2. Collectively, the present results suggested that the IHH signaling pathway may mediate the effect of cyclic stretch on the OLF cells.

\section{Introduction}

Ossification of the ligamentum flavum (OLF) is one of the degenerative changes to the ligamentum flavum (LF) that occur with aging (1). Since the first report of OLF in 1920, OLF has been investigated mainly in Asian countries (2). In China and Japan, the prevalence of OLF is 3.8\% (3) and $6.2 \%$ (4), respectively. OLF arises from the lateral capsular portion and exhibits continuity with the bony laminae (5). Clinical manifestations of OLF include regional thoracic stiffness and pain, which usually progresses to myelopathy (6). The diagnosis of OLF is often missed or delayed due to its slow progression and the co-occurrence of other spinal diseases $(5,6)$, which results in a heavy burden to both the patients and society.

OLF is a heterotopic ossification that occurs in the ligament $(5,6)$. OLF usually starts from the degeneration and hypertrophy of the elastic fibrous tissues, which results in the production of fibrocartilage-like cells and the proliferation of chondrocytes (7). Subsequently, chondrocytes are calcified, and with the growth of small vessels into the LF tissues, mature bone structures are formed (7). However, the etiology of OLF is not fully understood. Multiple factors have been implicated in the development of OLF, including genetics (8), systematic hormones (9), inflammatory markers (10) and 
mechanical stressors (11). Moreover, OLF is closely related to disturbance of the local mechanical microenvironment of the spine (12). The reduced stability of the middle and lower thoracic joints $(\mathrm{T})$ due to long-term rotational motions has been indicated the anatomical basis of OLF (13). A previous study reported T9-T12 are the common segments affected by OLF, suggesting that increases in local stress caused by longitudinal axial instability of the middle and lower thoracic spine may be the biomechanical basis of OLF (14).

In rats, after periodic mechanical stress stimulation, LF of the caudal vertebrae shows similar pathology to that found in humans with OLF: An extensive proliferation of the chondrocyte-like cells and ectopic cartilage formation near the insertion point of the LF (15). In addition, Cai et al (16) found that the expression levels of the ossification markers osteopontin $(O P N)$ and sex-determining region Y-box protein 9 (SOX9) are significantly upregulated after stress stimulation. In contrast, normal ligamentum cells did not show this significant upregulation in expression levels (16). Therefore, periodic mechanical stress can facilitate the progression of OLF at the cellular level.

Indian hedgehog $(I H H)$, a member of the secretory morphogen signaling family, is a secreted molecule that regulates cells via concentration gradients (17). The IHH signaling pathway is key for bone growth and development (18). IHH is one of the essential signaling pathways mediating heterotopic ossification of the extremities (19). Furthermore, the IHH pathway can directly or indirectly promote the proliferation and hypertrophy of the chondrocytes, induce the expression of parathyroid hormone-related peptide in cells surrounding the chondrocytes, facilitate the differentiation of resting chondrocytes into proliferating ones and induce differentiation of deep perichondrocytes into osteoblasts $(20,21)$. In an $\mathrm{IHH}^{-1-}$ mouse model, no deep perichondrocytes differentiation into osteoblasts is observed (22). Regard et al (23), found that the IHH pathway independently causes heterotopic ossification of the extremities; furthermore, inhibition of the IHH pathway significantly reduced the degree of heterotopic ossification. Thus, previous studies have indicated that the IHH signaling pathway may play a role in the process of bone development and heterotopic ossification. However, whether the $\mathrm{IHH}$ signaling pathway mediates osteogenic differentiation during ossification of LF requires further investigation.

Currently, there is no effective medication to prevent or delay the progress of OLF (5). Once ossification causes oppression to the spinal cord, the only treatment solution for OLF is surgery (5). However, OLF progression does not decrease or stop, despite this surgical intervention (24-26). Therefore, it is necessary to investigate the signaling pathways underlying the progression of OLF in order to understand the pathogenesis of OLF. The aim of the present study was to examine the involvement of the $\mathrm{IHH}$ signaling pathway in the development of OLF at the cellular and tissue levels, by simulating the in vivo stress environment of the LF. The present results will help in the understanding of the mechanisms underlying the development of OLF, and provide evidence for potential targets in novel therapeutic strategies.

\section{Materials and methods}

Patient specimens. The Ethics Committee from The Second Military Medical University approved the present study.
Participants provided written informed consent prior to specimen collection. The diagnosis of OLF was confirmed by clinical symptoms and radiological examinations. Patients were included if they received posterior open decompressive laminectomy between January 2016 and January 2019 at Changzheng Hospital, Second Military Medical University. A total of $18 \mathrm{LF}$ tissue samples (male patients, 10; female patients, 8; mean age, 61.2 years; age range, 52-73 years) from patients with OLF were obtained, of which 10 samples were harvested for cell culture. The remaining eight samples were used for histology. The non-ossified LF samples from 12 patients were used as controls (male patients, 7; female patients, 5; mean age, 56.2 years; age range, $42-68$ years), of which four samples were harvested for cell culture. The remaining eight samples were used for histology. Patients in the control group underwent posterior surgical procedures for disc herniation $(n=7)$ and fractures $(n=5)$. Thus, eight samples from the OLF group and eight samples from the control group were used for the tissue experiments. Whole pieces of ligaments were isolated and harvested after removing the lamina during the surgery. Patients who had congenital bone diseases or musculoligamentous tissue abnormalities were excluded.

Cell culture. A tissue explant method (16) was used to obtain the cultured LF cells. The LF and OLF tissues were obtained aseptically during surgery. For OLF tissues, the ossified areas were separated and removed under a microscope to avoid contamination with osteogenic cells. The LF and OLF ligaments were digested in $0.25 \%$ trypsin, followed by $250 \mathrm{U} /$ ml type I collagenase (Gibco; Thermo Fisher Scientific, Inc.). Subsequently, the fragments were washed with PBS and cultured at $37^{\circ} \mathrm{C}$ with $5 \% \mathrm{CO}_{2}$ in a $10-\mathrm{cm}$ dish with DMEM: Nutrient Mixture F-12 (Gibco; Thermo Fisher Scientific, Inc.) supplemented with 10\% FBS (Gibco; Thermo Fisher Scientific, Inc.) and $100 \mu \mathrm{g} / \mathrm{ml}$ penicillin/streptomycin. The cultures were left undisturbed for 2 days and then replaced with fresh medium. The cells, which were obtained from the explants, were treated with $0.25 \%$ trypsin containing $0.02 \%$ EDTA for $1-2 \mathrm{~min}$ at $37^{\circ} \mathrm{C}$, re-suspended and cultured for further passages. Cells at passage three were used for subsequent experiments.

Procedures of cyclic stretch application. A Flexcell FX-5000 strain unit (Flexcell International Corporation) was used in this study, with procedures similar to a previous study (16). Cells were cultured $\left(5 \times 10^{5}\right.$ cells/well) in a flexible bottomed polystyrene plate $(6$ wells $)$ with type I collagen $(0.15 \mathrm{mg} / \mathrm{ml})$ coated at the bottom (Flex I, Bioflex Plates; Flexcell International Corporation). After cell attachment, cyclic stretch was applied to the cells at $37^{\circ} \mathrm{C}$ for different durations $(0,6,12$ or $24 \mathrm{~h})$. Based on a previous study (16), the stretch parameter was set at a maximum $15 \%$ elongation. In the present study, the cultured cells were subjected to $15 \%$ elongation for $10 \mathrm{sec}$ and then relaxation for the next $10 \mathrm{sec}$. In pathway inhibition tests, subconfluent cells were pre-treated with $0.5 \mu \mathrm{M}$ Cyclopamine (Cpn; Sigma-Aldrich; Merck KGaA) for $2 \mathrm{~h}$ at $37^{\circ} \mathrm{C}$ and subsequently subjected to cyclic stretch for $24 \mathrm{~h}$ at $37^{\circ} \mathrm{C}$.

Reverse transcription-quantitative PCR (RT-qPCR). Total RNA was extracted from OLF and LF samples or cultured OLF cells using TRIzol ${ }^{\circledR}$ reagent (Invitrogen; Thermo Fisher 
Table I. Primers used for reverse transcription-quantitative PCR.

\begin{tabular}{|c|c|c|}
\hline Target & Primer sequence & Genbank accession \\
\hline$G A P D H$ & $\begin{array}{l}\text { F: 5'-AGGTCGGTGTGAACGGATTTG-3' } \\
\text { R: 5'-TGTAGACCATGTAGTTGAGGTCA-3' }\end{array}$ & NM_008084 \\
\hline$R U N X 2$ & $\begin{array}{l}\text { F: 5'-ATGCTTCATTCGCCTCACAAA-3' } \\
\text { R: 5'-GCACTCACTGACTCGGTTGG-3' }\end{array}$ & NM_001146038 \\
\hline Osterix & $\begin{array}{l}\text { F: 5'-ATGGCGTCCTCTCTGCTTG-3' } \\
\text { R: 5'-TGAAAGGTCAGCGTATGGCTT-3' }\end{array}$ & AF477981.1 \\
\hline$A L P$ & $\begin{array}{l}\text { F: 5'-CCAACTCTTTTGTGCCAGAGA-3' } \\
\text { R: 5'-GGCTACATTGGTGTTGAGCTTTT-3' }\end{array}$ & NM_007431 \\
\hline OCN & $\begin{array}{l}\text { F: 5'-CTGACCTCACAGATCCCAAGC-3' } \\
\text { R: 5'-TGGTCTGATAGCTCGTCACAAG-3' }\end{array}$ & X04142 \\
\hline$I H H$ & $\begin{array}{l}\text { F: 5'-CGGCTTTGACTGGGTGTATT-3' } \\
\text { R: 5'-AAAATGAGCACATCGCTGAA-3' }\end{array}$ & KR710697.1 \\
\hline$S M O$ & $\begin{array}{l}\text { F: 5'-CTGTCCTGCGTCATCATCTTT-3' } \\
\text { R: 5'-CCACAGCAAGGATTGCCAC-3' }\end{array}$ & NM_005631.5 \\
\hline GLII & $\begin{array}{l}\text { F: 5'-AAGCGTGAGCCTGAATCTGT-3' } \\
\text { R: 5'-CAGCATGTACTGGGCTTTGA-3' }\end{array}$ & BC013000.2 \\
\hline GLI2 & $\begin{array}{l}\text { F: 5'-CGACACCAGGAAGGAAGGTA-3' } \\
\text { R: 5'-TGCACAGAACGGAGGTAGTG-3' }\end{array}$ & BC172434.1 \\
\hline GLI3 & $\begin{array}{l}\text { F: 5'-CTTTGCAAGCCAGGAGAAAC-3' } \\
\text { R: 5'-TTGTTGGACTGTGTGCCATT-3' }\end{array}$ & BC113616.1 \\
\hline
\end{tabular}

F, forward; R, reverse; $A L P$, alkaline phosphatase; $R U N X 2$, Runt-related transcription factor 2; $O C N$, osteocalcin; $I H H$, Indian hedgehog; $S M O$, Smoothened; GLI, GLI family zinc finger.

Scientific, Inc.), according to the manufacturer's instructions. RNA concentration was then measured and converted to cDNA using a PrimerScript RT kit (Takara Bio, Inc.), according to the manufacturer's instructions. The mRNA expression levels of the osteogenic genes Runt-related transcription factor 2 (RUNX2), Osterix, alkaline phosphatase $(A L P)$ and osteocalcin $(O C N)$, and the IHH signaling pathway genes $I H H$, Smoothened (SMO), GLI family zinc finger 1 (GLI1), GLI2 and GLI3 were quantified using SYBR Premix Ex Taq ${ }^{\text {TM }}$ II (Takara Bio, Inc.). GAPDH was used as an endogenous control gene. The following qPCR conditions were used: Initial denaturation at $95^{\circ} \mathrm{C}$ for $10 \mathrm{~min}$; followed by 40 cycles of $95^{\circ} \mathrm{C}$ for $30 \mathrm{sec}$; and final extension at $60^{\circ} \mathrm{C}$ for $1 \mathrm{~min}$. The relative expression levels of each gene were calculated by the $2^{-\Delta \Delta \mathrm{Cq}}$ method (27). Primer sequences used for qPCR are presented in Table I.

Western blot analysis. Protein samples were extracted from the OLF cells using RIPA lysis buffer (Invitrogen; Thermo Fisher Scientific, Inc.), according to the manufacturer's instructions. Detailed procedures were described previously (28). The bicinchoninic acid protein assay (Pierce; Thermo Fisher Scientific, Inc.) was used to measure the protein concentration. The protein samples $(\sim 60 \mu \mathrm{g} / \mathrm{lane})$ were separated by electrophoresis using 10\% separating gel and 5\% stacking gel, and then transferred onto PVDF membranes. After blocking with skimmed milk in 5\% Tris-buffered saline with $0.05 \%$ Tween-20 for $1 \mathrm{~h}$ at room temperature, the membranes were subsequently incubated overnight at $4^{\circ} \mathrm{C}$ with primary antibodies targeted against: RUNX2 (cat. no. ab23981; Abcam; 1:1,000), Osterix (cat. no. ab22552; Abcam; 1:500), ALP (cat. no. ab67228; Abcam; 1:500), OCN (cat. no. ab93876; Abcam; 1:500), IHH (cat. no. ab39634; Abcam; 1:1,000), SMO (cat. no. ab113438; Abcam, 1:500) and GAPDH (cat. no. ab181602; Abcam; 1:5,000). The next day, membranes were incubated with a horseradish peroxide (HRP)-conjugated secondary antibody (cat. no. ab205718; Abcam; 1:2,000) for $1 \mathrm{~h}$ at room temperature and detected with electrochemiluminescence (GE Healthcare). Protein expression was quantified using Image $\mathbf{J}$ software (v1.51; National Institutes of Health) with GAPDH as the loading control.

Immunohistochemical analysis. Immunohistochemistry was performed in OLF and LF tissues following previously described (28). OLF and LF samples were fixed with $4 \%$ formaldehyde for $24 \mathrm{~h}$ at room temperature, embedded in paraffin and cut at $4-\mu \mathrm{m}$ thickness. The sections underwent antigen retrieval at $120^{\circ} \mathrm{C}$ for $10 \mathrm{~min}$ in a citrate solution $(10 \mathrm{mmol} / \mathrm{l}$; $\mathrm{pH}$ 6.0). After blocking with $1 \%$ bovine serum albumin (Gibco; Thermo Fisher Scientific, Inc.) for $37^{\circ} \mathrm{C}$ at $15 \mathrm{~min}$, the sections were subsequently incubated overnight at $4^{\circ} \mathrm{C}$ with the following primary antibodies: Rabbit polyclonal antibody against RUNX2 (cat. no. ab23981; Abcam; 1:500), Osterix (cat. no. ab22552; Abcam; 1:200), ALP (cat. no. ab224335; Abcam; 1:400), OCN (cat. no. ab93876; Abcam; 1:200), IHH 

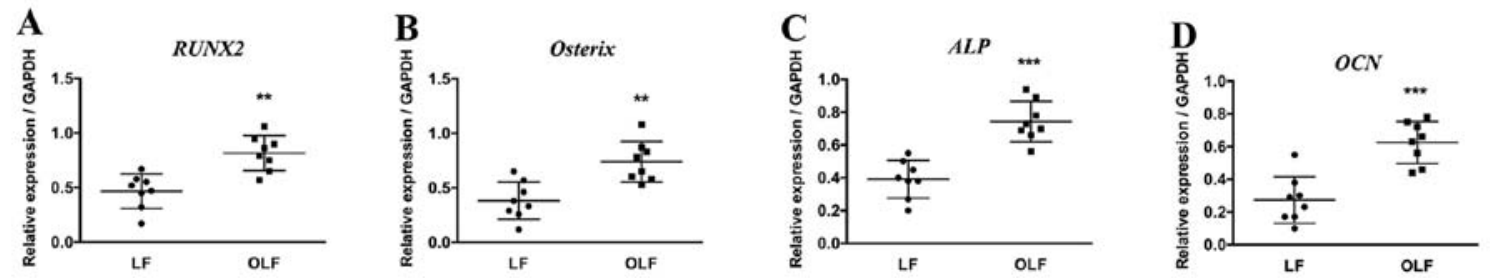

$\mathbf{E}$

RUNX2

G
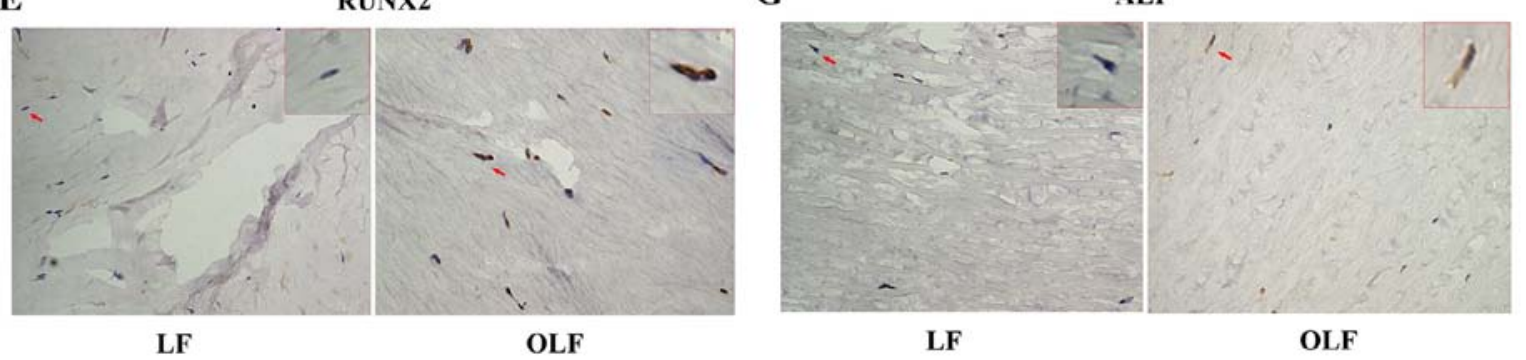

$\mathbf{F}$

Osterix

H
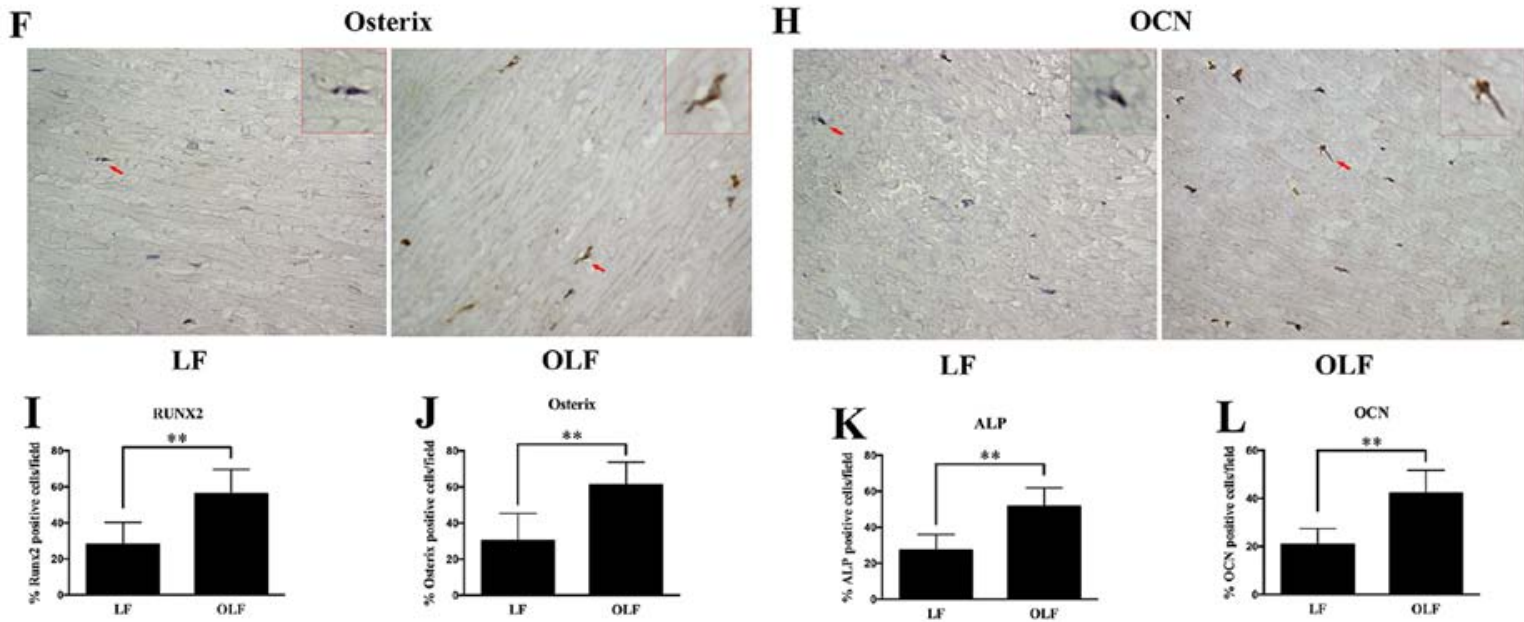

Figure 1. Expression of RUNX2, Osterix, $A L P$ and $O C N$ in LF and OLF tissues. Reverse transcription-quantitative PCR results for the mRNA expression levels of (A) RUNX2, (B) Osterix, (C) ALP and (D) OCN in the LF and OLF groups. Immunohistochemistry images of (E) RUNX2, (F) Osterix, (G) $A L P$ and (H) OCN. Quantification of the protein expression levels of (I) RUNX2, (J) Osterix, (K) ALP and (L) OCN in the LF group and OLF group. Magnification, $\mathrm{x} 400$. The red arrows represent the typical cells, which were magnified and shown in the upper right-hand corner (magnification, $\mathrm{x} 1,600)$. $\mathrm{N}=8 .{ }^{* *} \mathrm{P}<0.01$, ${ }^{* * * *} \mathrm{P}<0.001$ vs. LF samples. OLF, ossification of ligamentum flavum; LF, ligamentum flavum; RUNX2, Runt-related transcription factor 2; $A L P$, alkaline phosphatase; $O C N$, osteocalcin.

(cat. no. ab39634; Abcam; 1:200) and SMO (cat. no. ab113438; Abcam; 1:100). The next day, the sections were incubated with the HRP-conjugated secondary antibody (cat. no. ab205718; Abcam; $1: 2,000$ ) for $1 \mathrm{~h}$ at room temperature, followed by incubation with 3,3'-diaminobenzidine solution for 2-5 min at room temperature and counterstaining with hematoxylin at room temperature for $30 \mathrm{sec}-1 \mathrm{~min}$. Stained cells were identified using a ZEISS light microscope (magnification, x400; ZEISS Axio Imager A2; Carl Zeiss AG). Within each section, 200 cells were counted at random and the proportion of immunopositive cells was calculated (29). For quantitative analysis, images from three different sections of each sample were analyzed using Image $\mathbf{J}$ software (v1.51; National Institutes of Health).

Measurement of ALP activity and histochemical staining. ALP activity was quantitatively measured using an LabAssay ALP kit (cat. no. 291-58601; Wako Pure Chemical Industries, Ltd.) and histochemical staining was performed using a ALP color development kit (cat. no. C3206; Beyotime Institute of
Biotechnology) as previously described (30). Cell pellets were obtained by centrifugation at $3,000 \mathrm{xg}$ for $5 \mathrm{~min}$ at $4^{\circ} \mathrm{C}$. The total cellular protein was collected from the cultured cells by lysing cell pellets with RIPA lysis buffer (Invitrogen; Thermo Fisher Scientific, Inc.). The concentration of the protein was measured using the BCA method (Pierce; Thermo Fisher Scientific, Inc.). The protein solution was then analyzed for ALP activity using the ALP kit. The absorbance at $450 \mathrm{~nm}$ was measured using Spectra Max 250 spectrophotometer. The ALP-specific activity was determined using a standard curve and normalized to the protein concentration (U/mg). For histochemical staining, the cultured cells were washed with PBS and then fixed in $4 \%$ formaldehyde for $10 \mathrm{~min}$ at room temperature. The cells were stained with ALP reagent at $37^{\circ} \mathrm{C}$ for $30 \mathrm{~min}$, and ALP-positive cells were stained blue. Stained cells were identified using a ZEISS light microscope (magnification, x400; ZEISS Axio Imager A2; Carl Zeiss AG). In total, five random fields in each well were used for the analysis. ALP staining intensities were applied to evaluate the ability of osteogenic differentiation in OLF cells. 

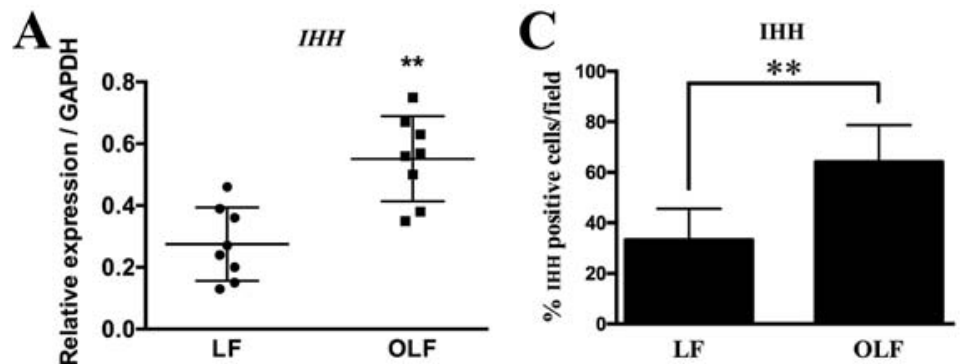

B

\section{IHH}
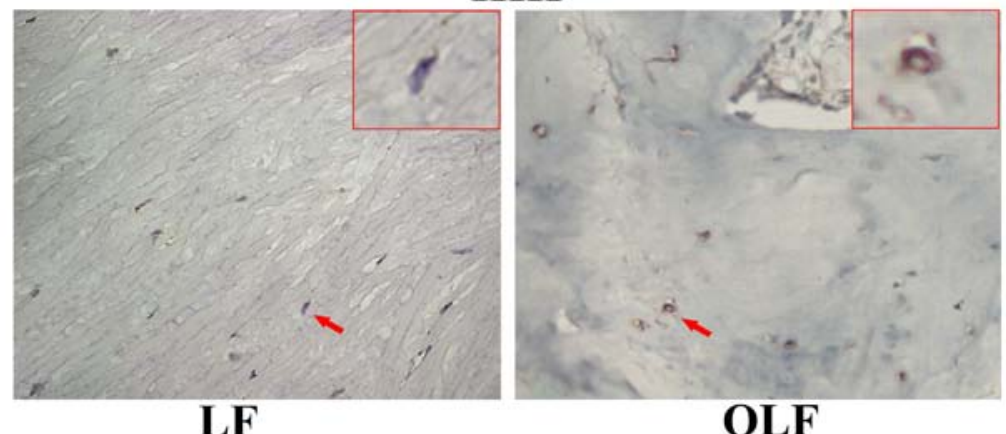

Figure 2. Expression level of $I H H$ in LF and OLF tissues. (A) Reverse transcription-quantitative PCR results showed the mRNA expression levels of $I H H$ between the LF group and OLF group. (B) Representative images of immunohistochemistry staining from each group. Magnification, $\mathrm{x} 400$. The red arrows represent the typical cells, which were magnified and shown in the upper right-hand corner (magnification, x1,600). (C) Immunohistochemistry results of the protein expression levels of $I H H$ in the $\mathrm{LF}$ group and OLF group. $\mathrm{N}=8 .{ }^{* *} \mathrm{P}<0.01 \mathrm{vs}$. LF samples. $I H H$, Indian hedgehog; OLF, ossification of ligamentum flavum; LF, ligamentum flavum.

Statistical analysis. Statistical analyses were performed using SPSS v19.0 (SPSS, Inc.). The experiments were repeated three times. Data are presented as the mean \pm SD. The results were analyzed with a Student's t-test, or one-way ANOVA followed by Tukey's post hoc test or Dunnett's test where appropriate. $\mathrm{P}<0.05$ was considered to indicate a statistically significant difference.

\section{Results}

Expression of osteogenic genes in OLF. RUNX2, ALP, Osterix and $O C N$ are important genes that define the osteogenesis process $(31,32)$. In the present study, the mRNA and protein expression levels of $R U N X 2$, Osterix, $A L P$ and $O C N$ were compared between the LF and OLF groups (Fig. 1). RT-qPCR and immunohistochemical analyses indicated that the OLF group had higher mRNA and protein expression levels of $R U N X 2$ compared with the LF group. Moreover, the OLF group had significantly higher mRNA and protein expression levels of Osterix, ALP and OCN compared with the LF group $(\mathrm{P}<0.01)$. Therefore, the present results suggested that the differentiation of fibroblasts into osteoblasts plays a role in OLF development.

Expression of IHH signaling genes in OLF. The mRNA and protein expression levels of $I H H$ in both groups are shown in Fig. 2. The RT-qPCR and immunohistochemical results revealed that the OLF group had significantly higher mRNA and protein expression levels of $I H H$ compared with the LF group $(\mathrm{P}<0.01)$. Thus, IHH may be involved in the osteogenesis of OLF.

Effects of cyclic stretch on osteogenic differentiation of $L F$ and OLF cells. The mRNA expression levels of osteogenic genes and IHH signaling genes in OLF and LF cells are shown in Fig. 3. It was demonstrated that application of cyclic stretch to OLF cells resulted in significant increases in mRNA expression levels of RUNX2, Osterix, ALP and $O C N$, and the IHH signaling genes $I H H$ and $S M O$ at $24 \mathrm{~h}$. Moreover, in LF cells the application of cyclic tensile strain significantly increased the mRNA expression level of $A L P$, but not of RUNX2, Osterix, OCN, IHH and SMO (Fig. 3).

To further examine the effect of mechanical stress on the osteogenesis of OLF cells, the expression levels of osteogenic genes were compared after OLF cells were treated with or without cyclic stretch for 6,12 and $24 \mathrm{~h}$. It was found that cyclic stretch caused a significant elevation in the mRNA expression levels of RUNX2, Osterix, ALP and $O C N$ at all time points (Fig. 4A-D). Furthermore, cyclic stretch was determined to significantly increase the protein expression levels of RUNX2, Osterix and $A L P$ at 6, 12 and $24 \mathrm{~h}$ (Fig. 4E-G). In addition, it was demonstrated that cyclic stretch caused a significant increase in the protein expression level of $O C N$ at 12 and $24 \mathrm{~h}$ (Fig. 4H). ALP activity was also significantly enhanced at 12 and $24 \mathrm{~h}$, with the greatest increase observed at $24 \mathrm{~h}$ (Fig. 4I and J). Collectively, the present results indicated that mechanical stress induced the osteogenesis process during the development of OLF.

Effects of cyclic stretch on the IHH signaling pathway. The IHH signaling pathway, which includes $I H H, S M O$ and GLI, is an important pathway in the osteogenic differentiation (33). To further examine the effect of local stress on the IHH signaling pathway and osteogenesis in OLF, cyclic stretch was applied to OLF cells for 6,12 and $24 \mathrm{~h}$. The relative ratios of $I H H, S M O$ and $G L I$ expression levels in OLF cells 

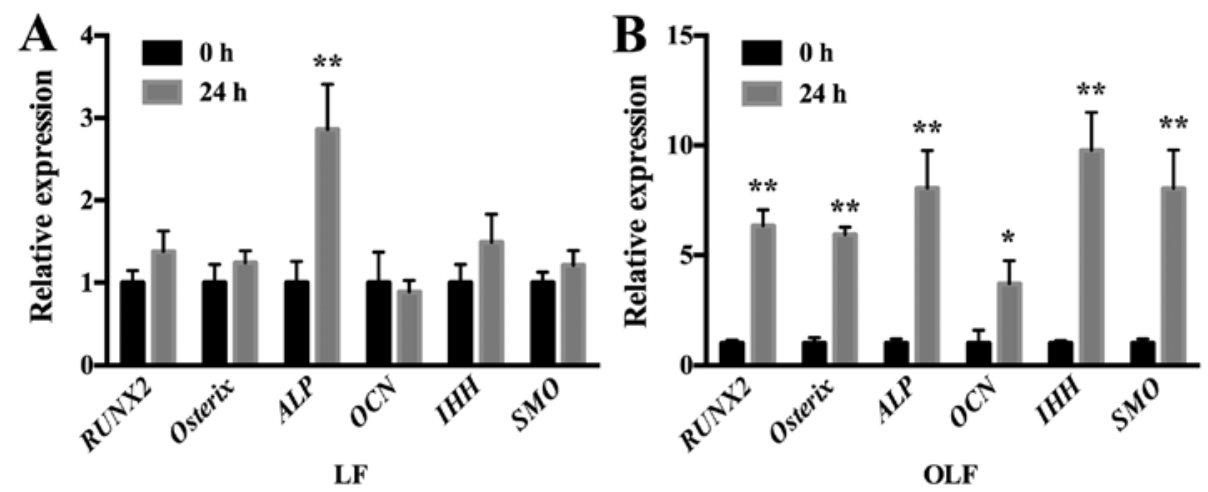

Figure 3. Expression level of osteogenic genes and $I H H$ signaling genes in LF and OLF cells after cyclic stretch. (A) Reverse transcription-quantitative PCR results showed that in LF cells, cyclic stretch increased the ALP expression level, while other gene expression levels did not change. (B) Relative mRNA expression levels of RUNX2, Osterix, ALP, OCN, IHH and SMO significantly increased after the application of cyclic stretch in OLF cells. ${ }^{\mathrm{P}} \mathrm{P}<0.05,{ }^{* *} \mathrm{P}<0.01$ vs. 0 h. Data are presented as the mean $\pm \mathrm{SD}$. N=3. OLF, ossification of ligamentum flavum; LF, ligamentum flavum; RUNX2, Runt-related transcription factor 2; ALP, alkaline phosphatase; $O C N$, osteocalcin; $I H H$, Indian hedgehog.

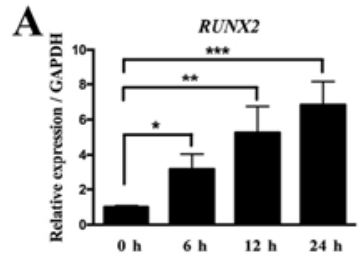

$\mathbf{E}$

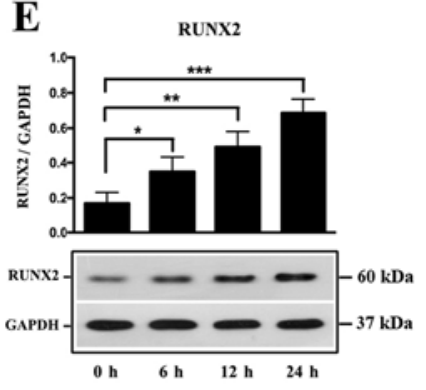

I

$\mathbf{0 h}$

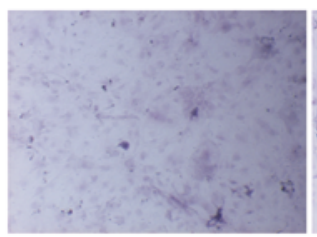

B

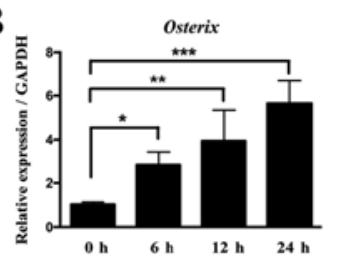

F

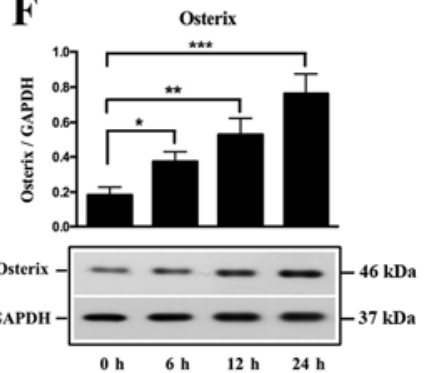

$12 \mathrm{~h}$

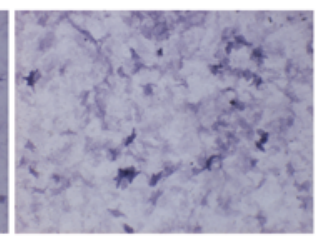

C

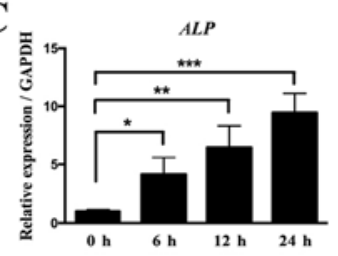

G
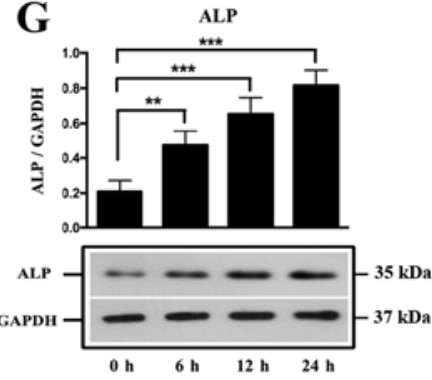

$24 \mathrm{~h}$

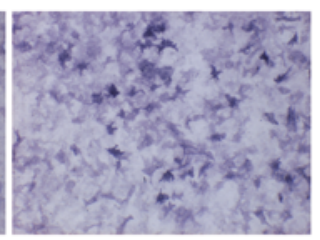

D

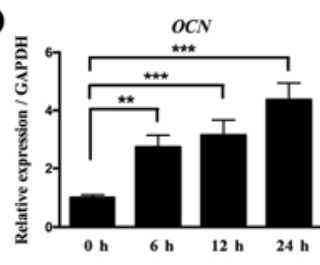

H

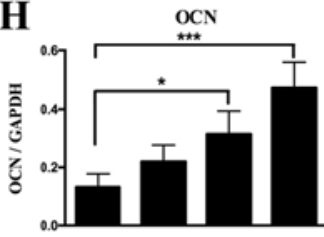

$6 \mathrm{~h}$

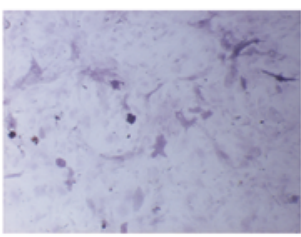

Figure 4. Effect of cyclic stretch on osteogenic genes and $A L P$ activity. Expression levels of (A) RUNX2, (B) Osterix, (C) $A L P$ and (D) $O C N$ in OLF cells were detected after cyclic stretch treatment for 0, 6, 12 and $24 \mathrm{~h}$. Protein expression levels of (E) RUNX2, (F) Osterix, (G) ALP and (H) OCN after cyclic stretch. (I) Representative images of $A L P$ staining. Magnification, $\mathrm{x} 400$. (J) Cyclic stretch increased $A L P$ activity in OLF cells. ${ }^{*} \mathrm{P}<0.05,{ }^{* *} \mathrm{P}<0.01,{ }^{* * *} \mathrm{P}<0.001$. Data are presented as the mean $\pm \mathrm{SD}$. N=3. OLF, ossification of ligamentum flavum; RUNX2, Runt-related transcription factor 2; $A L P$, alkaline phosphatase; $O C N$, osteocalcin.

are shown in Fig. 5. It was found that cyclic stretch significantly increased the mRNA and protein expression level of $I H H$ at 6,12 and $24 \mathrm{~h}$ (Fig. 5A and B). Moreover, the mRNA and protein expression levels of $S M O$ were significantly increased at all time points (Fig. 5C and D). Cyclic stretch also significantly upregulated the expression level of GLII at 6, 12 and $24 \mathrm{~h}$, and the mRNA expression level of GLI2 at 12 and $24 \mathrm{~h}$ (Fig. 5E and F); however, no significant effect on GLI3 expression level was detected (Fig. 5G). Therefore, the present results suggested that local stress could promote the expression of osteogenic markers by regulating the IHH signaling pathway in OLF cells.

IHH signaling is involved in osteogenesis induced by cyclic stretch. Cyclopamine (Cpn) can downregulate the IHH signaling pathway by binding to $S M O$ directly, which is a key component in IHH signaling (34). To examine whether cyclic stretch can directly affect the expression levels of RUNX2, Osterix, $A L P$ and $O C N$ via the IHH signaling pathway, OLF cells were treated with cyclic stretch alone or both cyclic stretch and Cpn. Fig. 6 

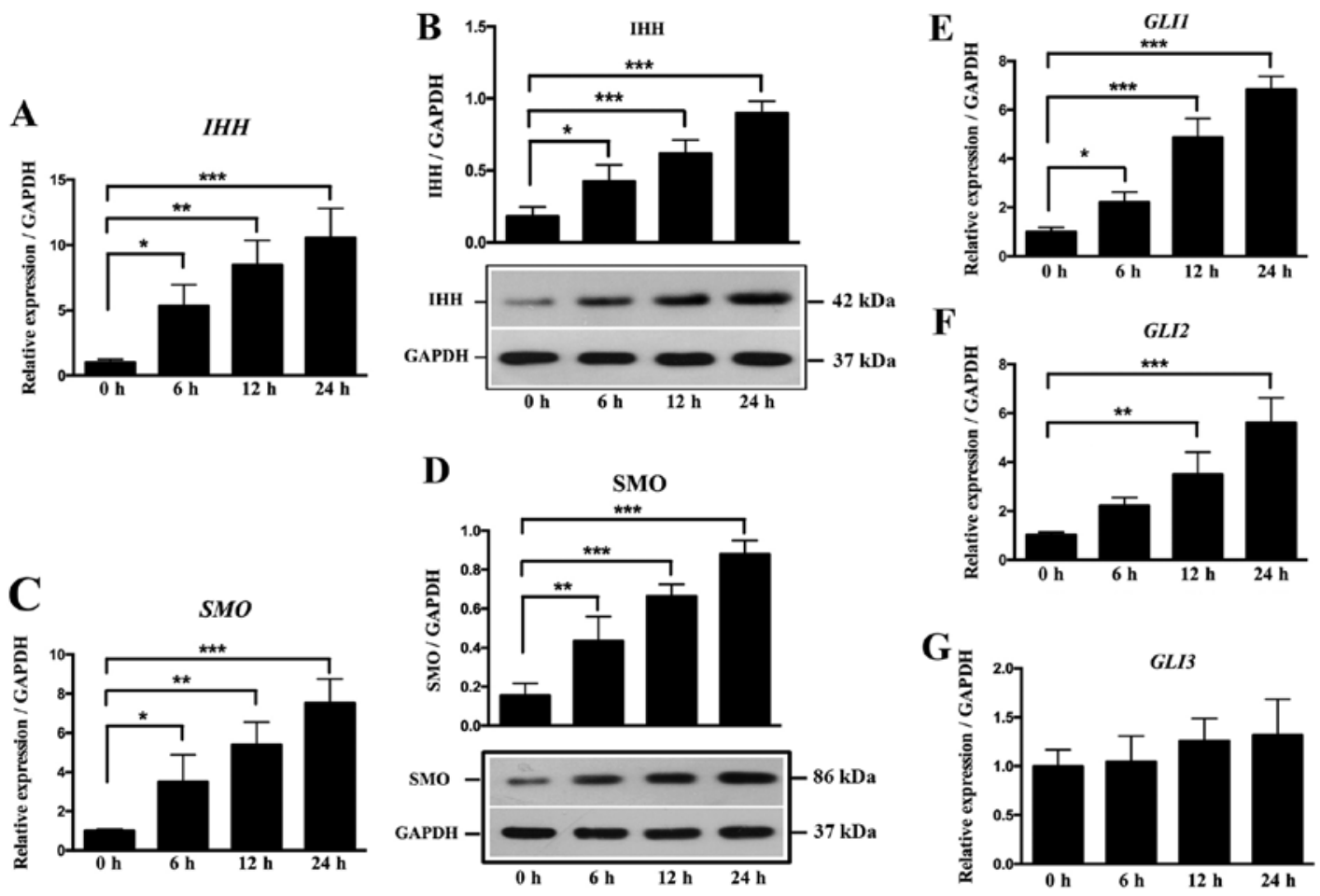

Figure 5. Effect of cyclic stretch on the expression of molecules involved in the $I H H$ signaling pathway in OLF cells. Cyclic stretch of OLF cells resulted in significant increases in the (A) mRNA and (B) protein expression levels of $I H H$. Cyclic stretch of OLF cells resulted in significant increases in the (C) mRNA and (D) protein expression levels of $S M O$ at 6,12 and $24 \mathrm{~h}$. mRNA expression levels of (E) GLI1 and (F) GLI2 were significantly increased after cyclic stretch. (G) No significant effect was observed on the expression level of GLI3. ${ }^{*} \mathrm{P}<0.05,{ }^{* *} \mathrm{P}<0.01,{ }^{* * * *} \mathrm{P}<0.001$. Data are presented as the mean $\pm \mathrm{SD}$. N=3. OLF, ossification of ligamentum flavum; $R U N X 2$, Runt-related transcription factor 2; $A L P$, alkaline phosphatase; $O C N$, osteocalcin; $I H H$, Indian hedgehog; $S M O$, Smoothened; GLI, GLI family zinc finger.
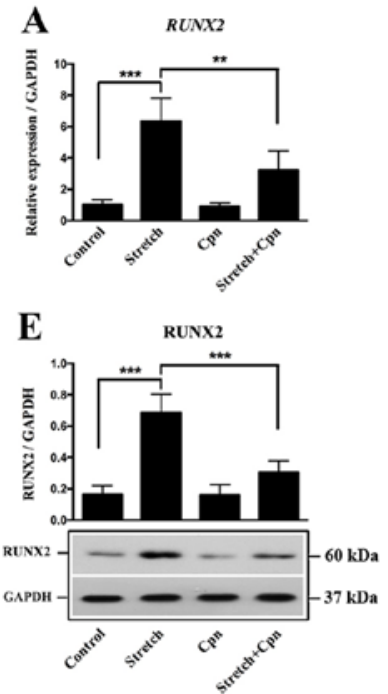

I Control

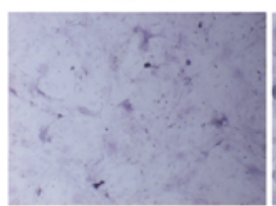

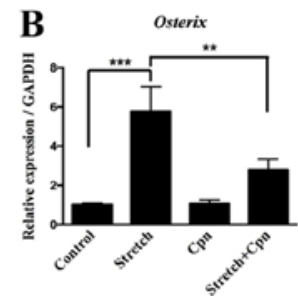

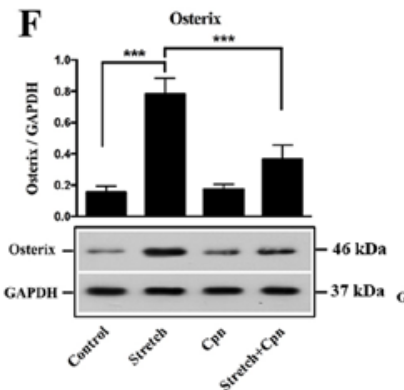

Stretch

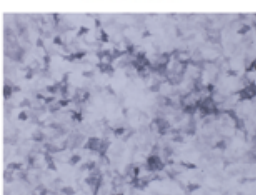

Cpn
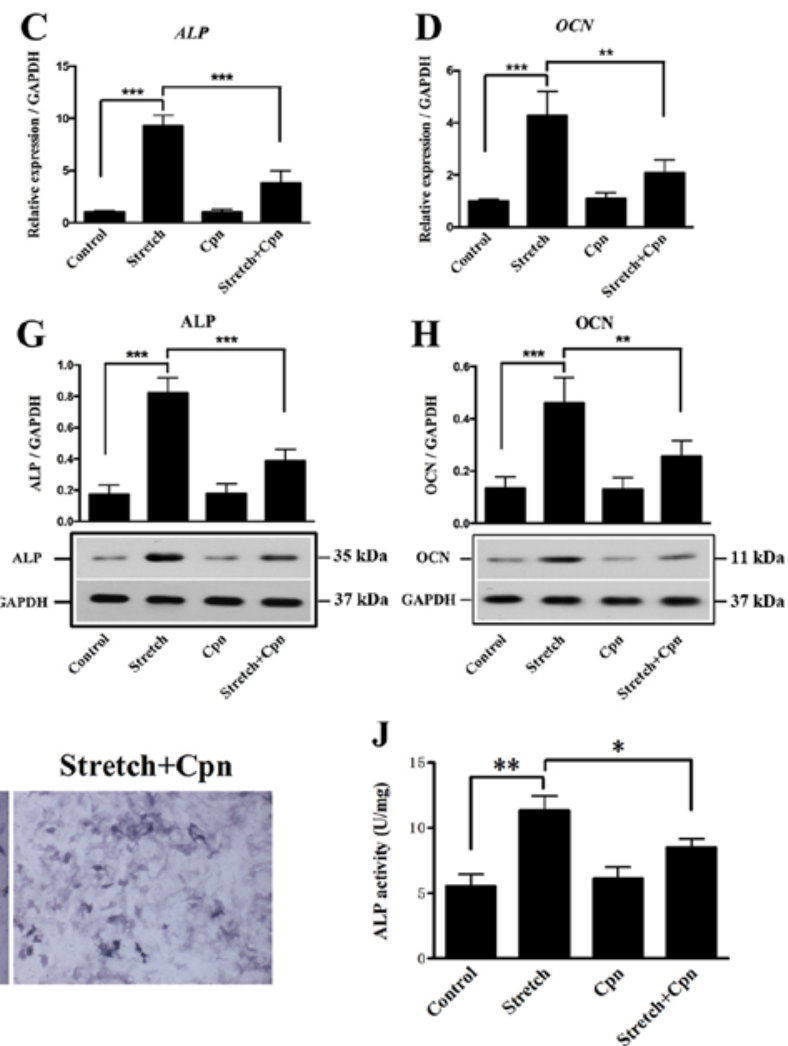

Figure 6. Effect of the Indian hedgehog signaling pathway inhibitor $\mathrm{Cpn}$ on the expression levels of RUNX2, Osterix, ALP and $O C N$ in OLF cells. mRNA expression levels of (A) RUNX2, (B) Osterix, (C) $A L P$ and (D) $O C N$. Protein expression levels of (E) RUNX2, (F) Osterix, (G) $A L P$ (H) and $O C N$ were significantly decreased in the stretch + Cpn group compared with cyclic stretch alone. (I) Representative images of $A L P$ staining. Magnification, $\mathrm{x} 400$. (J) $A L P$ activity was significantly reduced in the $\mathrm{Cpn}+$ stretch group compared with cyclic stretch alone. ${ }^{*} \mathrm{P}<0.05,{ }^{* *} \mathrm{P}<0.01,{ }^{* * *} \mathrm{P}<0.001$. Data are presented as the mean $\pm \mathrm{SD}$. N=3. OLF, ossification of ligamentum flavum; $R U N X 2$, Runt-related transcription factor 2; $A L P$, alkaline phosphatase; $O C N$, osteocalcin; Cpn, cyclopamine. 

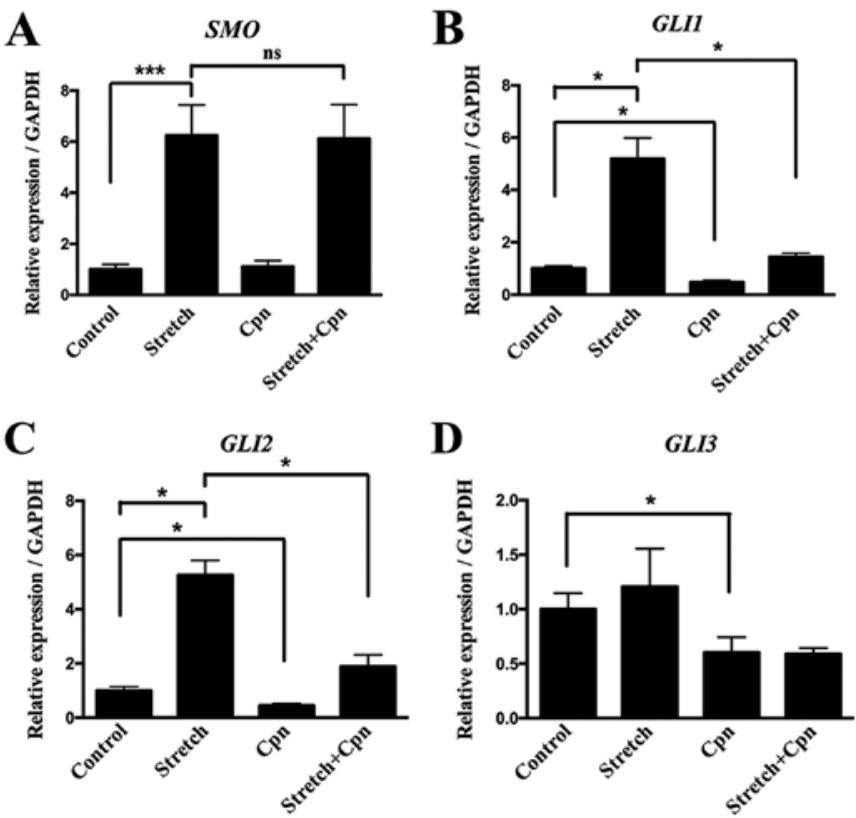

Figure 7. Effect of Cpn on the expression levels of Indian hedgehog signalingrelated genes in OLF cells. mRNA expression levels of (A) SMO, (B) GLII, (C) GLI2 and (D) GLI3. ${ }^{*} \mathrm{P}<0.05,{ }^{* * *} \mathrm{P}<0.001$. Data are presented as the mean \pm SD. $N=3$. OLF, ossification of ligamentum flavum; Cpn, cyclopamine; ns, not significant; SMO, Smoothened; GLI, GLI family zinc finger.

showed the expression levels of RUNX2, Osterix, ALP and $O C N$ after Cpn treatment. It was found that the expression levels of all genes were significantly decreased in the Cpn + cyclic stretch group compared with the cyclic stretch alone group (Fig. 6A-H). Furthermore, ALP activity was reduced with Cpn treatment compared with cyclic stretch alone (Fig. 6I and J). It was demonstrated that cells treated with cyclic stretch showed a significant increase in the expression levels of the downstream IHH signaling molecules SMO, GLII and GLI2. However, cyclic stretch did not significantly alter the expression of GLI3 compared with the control group. Moreover, Cpn alone decreased the expression of GLII, GLI2 and GLI3 compared with the control group. The effect of cyclic stretch on the expression of GLII and GLI2 was decreased by the addition of Cpn (Fig. 7). Thus, the present results provided evidence of the role of the IHH signaling pathway in the pathogenesis of OLF.

\section{Discussion}

OLF in East Asian populations is more common compared with individuals from other regions, and is characterized by pathological heterotopic ossification in the LF $(5,6)$. Various genetic, mechanical and inflammatory factors are involved in the development OLF (35), but the molecular mechanistic pathways remain to be elucidated. IHH signaling is an essential signaling pathway mediating heterotopic ossification of the extremities (19). To the best of our knowledge, the present study is one of the first to examined whether IHH signaling mediates osteogenic differentiation during OLF. It was found that the expression levels of osteoblastic markers and $I H H$ were higher in OLF tissues compared with in LF tissues. Moreover, cyclic stretch elevated the expression levels of osteogenic differentiation markers in the LF cells of patients with OLF.
Furthermore, it was demonstrated that osteogenic induction was attenuated by inhibiting IHH signaling. Collectively, the present results suggested that the IHH signaling pathway may play a role in the pathogenesis of OLF.

The development of OLF involves chondrogenic and osteogenic differentiation of the fibroblasts into osteoblasts (7). Therefore, examining osteogenic differentiation of the LF may help to understand the pathogenesis of OLF. Previous studies have identified higher expression levels of osteogenesis-related genes in the OLF cells and tissues (16,36). Using RNA sequencing, Yang et al (36), examined gene expression profiles of LF cells in both immature and mature ossification groups. Moreover, Yang et al (36) found that 42 osteogenesis-related factors were differentially expressed, of which the expression levels of SOX11, RUNX2, Osterix and secreted phosphoprotein 1 were increased. Cai et al (16), performed immunohistochemistry in the decalcified paraffin OLF sections, and found that $\beta$-catenin and SOX9 were immunopositive in premature chondrocytes. In addition, Cai et al (16) identified that the expression of $R U N X 2$ and $O P N$ was significantly increased in hypertrophic chondrocytes surrounding the calcification front. According to a previous in vitro study (37), cultured OLF cells have higher mRNA expression levels of $\beta$-catenin, RUNX2, SOX 9 and $O P N$ compared with non-OLF cells. In line with these previous results, the present study found that the expression levels of the osteoblastic markers RUNX2, Osterix, ALP and $O C N$ were higher in OLF tissues compared with LF tissues. Thus, the process of fibroblasts differentiating into osteoblasts may be an important physiopathological mechanism in OLF.

Mechanical and genetic factors may facilitate the development of OLF (5). Previous studies have demonstrated that the cyclic stretch could initiate and promote the ossification process by modulating the osteogenic differentiation of the spinal ligament cells $(15,38)$. The present study applied cyclic stretch to OLF cells, and found that both ALP activity and the expression levels of RUNX2, Osterix, ALP and $O C N$ were elevated as the duration of the stretch force was increased; these results were in line with previous results $(11,39)$. Ning et al (39), showed that cyclic mechanical application produces higher ALP activity and expression levels of $A L P$, $B M P 2, O P N$ and Osterix in the multiple-level OLF group compared with the single-level group. Furthermore, a previous in vitro study revealed that mechanical stress significantly increases the mRNA expression levels of $R U N X 2, A L P$ and $O C N$ in OLF cells compared with the control group (11).

The present results indicated that cyclic stretch increased the expression levels of IHH, SMO, GLII and GLI2 genes, which are involved in the IHH signaling pathway, at different time points. Furthermore, the present study blocked the IHH signaling pathway by treating OLF cells with Cpn, which is a potent inhibitor of the IHH signaling pathway (40). It was found that the impact of cyclic stretch on the expression levels of osteogenic genes was significantly attenuated after Cpn treatment. Thus, the present results suggested that cyclic stretch may affect osteogenic differentiation of OLF cells via the IHH signaling pathway. IHH signaling plays a critical role in prenatal endochondral bone formation and limb development, as well as postnatal bone formation $(18,41)$. Therefore, the complete ablation of IHH signaling can lead to the closure of the growth 
plate and immature bone growth $(18,41)$. IHH signaling may also participate in the bone repair process after a fracture (42). Furthermore, abnormal bone development and ectopic bone formation due to overexpression of the IHH signaling have been previously reported $(22,43)$. In the present study, the expression of $I H H, S M O, G L I I$ and GLI2 was upregulated by cyclic stretch, suggesting the IHH signaling pathway was involved in the pathogenesis of OLF. By contrast, it has been shown that the upregulation of patched 1 (PTC1) can decrease IHH signaling function (44). Moreover, it has been suggested that several IHH molecules can interact with the increased PTC1, resulting in reduced $S M O$ release (45). However, the present study did not identify any changes in the expression level of GLI3 under cyclic stretch conditions. Thus, GLI3 may not play a critical role in the regulation of osteogenic differentiation of OLF cells.

There are certain limitations to the present study. First, only the IHH signaling pathway was investigated, and other signaling pathways are likely involved in the differentiation of OLF cells. A previous study (16) demonstrated that cyclic stretch significantly elevated the expression levels of $\beta$-catenin, $R U N X 2, S O X 9$ and $O P N$, which indicates that the $\beta$-catenin signaling pathway may be involved in the pathogenesis of OLF. Second, the present study was conducted in vitro. Therefore, in vivo OLF models are required to assess the therapeutic effect of inhibiting IHH signaling on the progression of OLF. In addition, only the effects of mechanical stress on osteogenic differentiation were investigated, and other types of stress, such as compression and shear force, were not examined. Thus, further studies are required to investigate the specific mechanisms and therapeutic targets for OLF.

In conclusion, the present results suggested that the expression levels of osteogenic markers and IHH signaling genes were higher in OLF cells. Moreover, inhibition of the IHH signaling pathway reduced the expression levels of osteogenic markers. Thus, the IHH signaling pathway may be involved in the progression of OLF. If cell differentiation can be regulated by modulating this pathway, the homeostatic balance of cell differentiation could be maintained and the ossification of spinal ligaments may be prevented..

\section{Acknowledgements}

Not applicable.

\section{Funding}

The present study was supported by the National Natural Science Foundation of China (grant nos. 81501917 and 81802120) and the Shanghai Sailing Program (grant no. 18YF1423100).

\section{Availability of data and materials}

The datasets used and/or analyzed during the current study are available from the corresponding author on reasonable request.

\section{Authors' contributions}

RG and CS performed experiments, analyzed data and prepared the manuscript. CY and YZ analyzed and interpreted the data, and prepared the manuscript. $\mathrm{XC}$ and $\mathrm{XZ}$ designed experiments, analyzed data and finalized the manuscript. All authors read and approved the final manuscript.

\section{Ethics approval and consent to participate}

The study was approved by the Ethics Committee of Second Military Medical University, and the experimental procedures were performed in accordance with the standard guidelines. Informed consent was obtained from the patients or relatives for the collection of LF during surgery, and the use of these samples for scientific research.

\section{Patient consent for publication}

Not applicable.

\section{Competing interests}

The authors declare that they have no competing interests.

\section{References}

1. Nouri A, Tetreault L, Singh A, Karadimas SK and Fehlings MG: Degenerative cervical myelopathy: Epidemiology, genetics, and pathogenesis. Spine 40: E675-E693, 2015.

2. Takahashi T, Hanakita J and Minami M: Pathophysiology of calcification and ossification of the ligamentum flavum in the cervical spine. Neurosurg Clin N Am 29: 47-54, 2018.

3. Guo JJ, Luk KDK, Karppinen J, Yang H and Cheung KMC: Prevalence, distribution, and morphology of ossification of the ligamentum flavum: A population study of one thousand seven hundred thirty-six magnetic resonance imaging scans. Spine 35: $51-56,2010$

4. Kawaguchi Y, Yasuda T, Seki S, Nakano M, Kanamori M, Sumi S and Kimura T: Variables affecting postsurgical prognosis of thoracic myelopathy caused by ossification of the ligamentum flavum. Spine J 13: 1095-1107, 2013.

5. Ahn DK, Lee S, Moon SH, Boo KH, Chang BK and Lee JI: Ossification of the ligamentum flavum. Asian Spine J 8: 89-96, 2014.

6. Hirabayashi S: Ossification of the ligamentum flavum. Spine Surg Relat Res 1: 158-163, 2017.

7. Yayama T, Uchida K, Kobayashi S, Kokubo Y, Sato R, Nakajima $\mathrm{H}$, Takamura $\mathrm{T}$, Bangirana $\mathrm{A}$, Itoh $\mathrm{H}$ and Baba $\mathrm{H}$ : Thoracic ossification of the human ligamentum flavum: Histopathological and immunohistochemical findings around the ossified lesion. J Neurosurg Spine 7: 184-193, 2007.

8. Qu X, Chen Z, Fan D, Xiang S, Sun C, Zeng Y, Li W, Guo Z, Qi Q, Zhong W, et al: Two novel BMP-2 variants identified in patients with thoracic ossification of the ligamentum flavum. Eur J Hum Genet 25: 565-571, 2017.

9. Li H, Jiang LS and Dai LY: Hormones and growth factors in the pathogenesis of spinal ligament ossification. Eur Spine $\mathrm{J}$ 16: 1075-1084, 2007.

10. Zhang C, Chen Z, Meng X, Li M, Zhang L and Huang A: The involvement and possible mechanism of pro-inflammatory tumor necrosis factor alpha (TNF- $\alpha$ ) in thoracic ossification of the ligamentum flavum. PLoS One 12: e0178986, 2017.

11. Shunzhi Y, Zhonghai L and Ning Y: Mechanical stress affects the osteogenic differentiation of human ligamentum flavum cells via the BMP-Smad1 signaling pathway. Mol Med Rep 16: 76927698, 2017.

12. Edwards DS and Clasper JC: Heterotopic ossification: A systematic review. J R Army Med Corps 161: 315-321, 2015.

13. Kłosiński M, Skrzat J, Walocha J and Mizia E: Contemporary views on the ossification of the ligamenta flava. Ortop Traumatol Rehabil 14: 495-503, 2012.

14. Gao R, Yuan W, Yang L, Shi G and Jia L: Clinical features and surgical outcomes of patients with thoracic myelopathy caused by multilevel ossification of the ligamentum flavum. Spine $\mathbf{J} 13$ : 1032-1038, 2013 
15. Tsukamoto N, Maeda T, Miura H, Jingushi S, Hosokawa A, Harimaya K, Higaki H, Kurata K and Iwamoto Y: Repetitive tensile stress to rat caudal vertebrae inducing cartilage formation in the spinal ligaments: A possible role of mechanical stress in the development of ossification of the spinal ligaments. J Neurosurg Spine 5: 234-242, 2006.

16. Cai HX, Yayama T, Uchida K, Nakajima H, Sugita D, Guerrero AR, Yoshida A and Baba H: Cyclic tensile strain facilitates the ossification of ligamentum flavum through $\beta$-catenin signaling pathway: In vitro analysis. Spine 37: E639-E646, 2012.

17. Gallet A: Hedgehog morphogen: From secretion to reception. Trends Cell Biol 21: 238-246, 2011.

18. Yang J, Andre P, Ye L and Yang YZ: The Hedgehog signalling pathway in bone formation. Int J Oral Sci 7: 73-79, 2015.

19. Kan C, Chen L, Hu Y, Ding N, Lu H, Li Y, Kessler JA and Kan L: Conserved signaling pathways underlying heterotopic ossification. Bone 109: 43-48, 2018.

20. Minina E, Wenzel HM, Kreschel C, Karp S, Gaffield W, McMahon AP and Vortkamp A: BMP and Ihh/PTHrP signaling interact to coordinate chondrocyte proliferation and differentiation. Development 128: 4523-4534, 2001

21. Wongdee K, Thonapan N, Saengamnart W, Krishnamra N and Charoenphandhu N: Bromocriptine modulates the expression of PTHrP receptor, Indian hedgehog, and Runx 2 proteins in the growth plate of lactating rats. Mol Cell Biochem 381: 191-199, 2013.

22. St-Jacques B, Hammerschmidt $M$ and McMahon AP: Indian hedgehog signaling regulates proliferation and differentiation of chondrocytes and is essential for bone formation. Genes Dev 13: 2072-2086, 1999.

23. Regard JB, Malhotra D, Gvozdenovic-Jeremic J, Josey M, Chen M, Weinstein LS, Lu J, Shore EM, Kaplan FS and Yang Y: Activation of Hedgehog signaling by loss of GNAS causes heterotopic ossification. Nat Med 19: 1505-1512, 2013

24. Sugita S, Chikuda H, Takeshita K, Seichi A and Tanaka S: Progression of ossification of the posterior longitudinal ligament of the thoracic spine following posterior decompression and stabilization. J Neurosurg Spine 21: 773-777, 2014

25. Ando K, Imagama S, Ito Z, Kobayashi K, Ukai J, Muramoto A Shinjo R, Matsumoto $\mathrm{T}$, Nakashima $\mathrm{H}$ and Ishiguro $\mathrm{N}$ : Progressive relapse of ligamentum flavum ossification following decompressive surgery. Asian Spine J 8: 835-839, 2014.

26. Tokuhashi Y, Ajiro Y and Umezawa N: A patient with two re-surgeries for delayed myelopathy due to progression of ossification of the posterior longitudinal ligaments after cervical laminoplasty. Spine 34: E101-E105, 2009.

27. Livak KJ and Schmittgen TD: Analysis of relative gene expression data using real-time quantitative PCR and the 2(-Delta Delta C(T)) Method. Methods 25: 402-408, 2001.

28. Shi C, Wu H, Du D, Im HJ,Zhang Y, Hu B, Chen H, Wang X, Liu Y, Cao $\mathrm{P}$, et al: Nicotinamide phosphoribosyltransferase inhibitor APO866 prevents IL-1 $\beta$-induced human nucleus pulposus cell degeneration via autophagy. Cell Physiol Biochem 49: $2463-$ 2482,2018

29. Hu B, Shi C, Xu C, Cao P, Tian Y, Zhang Y, Deng L, Chen H and Yuan W: Heme oxygenase-1 attenuates IL-1 $\beta$ induced alteration of anabolic and catabolic activities in intervertebral disc degeneration. Sci Rep 6: 21190, 2016.

30. Du D, Zhou Z, Zhu L, Hu X, Lu J, Shi C, Chen F and Chen A: TNF- $\alpha$ suppresses osteogenic differentiation of MSCs by accelerating P2Y2 receptor in estrogen-deficiency induced osteoporosis. Bone 117: 161-170, 2018.
31. Krane SM: Identifying genes that regulate bone remodeling as potential therapeutic targets. J Exp Med 201: 841-843, 2005.

32. Karsenty G: The genetic transformation of bone biology. Genes Dev 13: 3037-3051, 1999.

33. Shimoyama A, Wada M, Ikeda F, Hata K, Matsubara T, Nifuji A, Noda M, Amano K, Yamaguchi A, Nishimura R, et al: Ihh/ Gli2 signaling promotes osteoblast differentiation by regulating Runx2 expression and function. Mol Biol Cell 18: 2411-2418, 2007.

34. Gould A and Missailidis S: Targeting the hedgehog pathway: The development of cyclopamine and the development of anti-cancer drugs targeting the hedgehog pathway. Mini Rev Med Chem 11: 200-213, 2011.

35. Uchida K, Yayama T, Cai HX, Nakajima H, Sugita D, Guerrero AR, Kobayashi S, Yoshida A, Chen KB and Baba H: Ossification process involving the human thoracic ligamentum flavum: Role of transcription factors. Arthritis Res Ther 13: R144, 2011.

36. Yang X, Chen Z, Meng X, Sun C, Li M, Shu L, Fan D, Fan T, Huang AY and Zhang C: Angiopoietin-2 promotes osteogenic differentiation of thoracic ligamentum flavum cells via modulating the Notch signaling pathway. PLoS One 13: e0209300, 2018.

37. Park JO, Lee BH, Kang YM, Kim TH, Yoon JY, Kim H, Kwon UH, Lee KI, Lee HM and Moon SH: Inflammatory cytokines induce fibrosis and ossification of human ligamentum flavum cells. J Spinal Disord Tech 26: E6-E12, 2013.

38. Zuscik MJ, Hilton MJ, Zhang X, Chen D and O'Keefe RJ: Regulation of chondrogenesis and chondrocyte differentiation by stress. J Clin Invest 118: 429-438, 2008

39. Ning S, Chen Z, Fan D, Sun C, Zhang C, Zeng Y, Li W, Hou X, $\mathrm{Qu} X$, Ma Y, et al: Genetic differences in osteogenic differentiation potency in the thoracic ossification of the ligamentum flavum under cyclic mechanical stress. Int J Mol Med 39: 135-143, 2017.

40. Incardona JP, Gaffield W, Kapur RP and Roelink H: The teratogenic Veratrum alkaloid cyclopamine inhibits sonic hedgehog signal transduction. Development 125: 3553-3562, 1998.

41. Ohba S: Hedgehog Signaling in endochondral ossification. J Dev Biol 4: E20, 2016.

42. Shi Y, He G, Lee WC, McKenzie JA, Silva MJ and Long F: Gli1 identifies osteogenic progenitors for bone formation and fracture repair. Nat Commun 8: 2043, 2017.

43. Day TF and Yang Y: Wnt and hedgehog signaling pathways in bone development. J Bone Joint Surg Am 90 (Suppl 1): 19-24, 2008.

44. Quijada L, Callejo A, Torroja C and Guerrero I: The patched receptor. In: Hedgehog-Gli Signaling in Human Disease. Boston, MA. Ruiz i Altaba A (ed). Springer, US, pp23-33, 2006.

45. Salem O, Wang HT, Alaseem AM, Ciobanu O, Hadjab I, Gawri R, Antoniou J and Mwale F: Naproxen affects osteogenesis of human mesenchymal stem cells via regulation of Indian hedgehog signaling molecules. Arthritis Res Ther 16: R152, 2014.

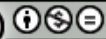

This work is licensed under a Creative Commons Attribution-NonCommercial-NoDerivatives 4.0 International (CC BY-NC-ND 4.0) License. 